\title{
Rosiglitazone reduces breast cancer risk in Taiwanese female patients with type 2 diabetes mellitus
}

\author{
Chin-Hsiao Tseng ${ }^{1,2,3}$ \\ ${ }^{1}$ Department of Internal Medicine, National Taiwan University College of Medicine, Taipei, Taiwan \\ ${ }^{2}$ Division of Endocrinology and Metabolism, Department of Internal Medicine, National Taiwan University Hospital, Taipei, \\ Taiwan \\ ${ }^{3}$ Division of Environmental Health and Occupational Medicine of the National Health Research Institutes, Zhunan, Taiwan
}

Correspondence to: Chin-Hsiao Tseng, email: ccktsh@ms6.hinet.net

Keywords: breast cancer, diabetes mellitus, rosiglitazone, Taiwan

Received: October 09, $2016 \quad$ Accepted: November 30, $2016 \quad$ Published: December 08, 2016

\section{ABSTRACT}

This study investigated whether rosiglitazone may affect breast cancer risk in female patients with type 2 diabetes mellitus in Taiwan. The reimbursement database of all female patients with type 2 diabetes mellitus under oral antidiabetic agents or insulin from 1996 to 2009 was retrieved from the National Health Insurance. An entry

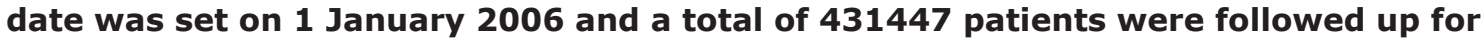
breast cancer incidence till the end of 2009. Incidences for ever users, never users and subgroups of rosiglitazone dose-response parameters (tertile cutoffs of cumulative duration and cumulative dose) were calculated and hazard ratios estimated by Cox regression. There were 53029 ever users and $\mathbf{3 7 8 4 1 8}$ never users, respective numbers of incident breast cancer $410(0.77 \%)$ and $3292(0.87 \%)$, and respective incidence 217.53 and 249.12 per 100000 person-years. The overall hazard ratio was 0.889 (95\% confidence interval: 0.797-0.992) in the fully adjusted model. Significantly lower risk was observed for the third tertiles of cumulative duration ( $>14$ months) and cumulative dose (> $1792 \mathrm{mg}$ ) while compared to never users, with respective adjusted hazard ratio of 0.815 (95\% confidence interval: $0.682-0.973)$ and 0.815 (95\% confidence interval: 0.682-0.974). Additionally, a significant interaction between metformin and rosiglitazone was observed. The lowest risk was seen in patients who used both drugs (hazard ratio 0.812, 95\% confidence interval: 0.705-0.934). In conclusion, rosiglitazone reduces breast cancer risk in female patients with type 2 diabetes mellitus, which shows a significant interaction with metformin.

\section{INTRODUCTION}

Rosiglitazone shows antiproliferative and apoptotic actions on breast cancer cells; and it may induce autophagy and inhibit the invasiveness and metastasis in breast cancer cell lines $[1,2]$. In rats treated with the carcinogen 7,12-dimethylbenz(a)anthracene, rosiglitazone suppresses mammary tumor growth [3]. A recent study found that rosiglitazone may inhibit breast cancer growth in mice by suppressing the expression of a pro-inflammatory and protumor protein Gpr132 in tumor-associated macrophages [4].

Whether these beneficial effects of rosiglitazone on breast cancer observed in cellular and animal studies can be extended to diabetic patients who use rosiglitazone as a therapeutic agent remains to be explored. In a pilot short-term clinical study conducted in women with newly diagnosed early breast cancer (stage $0-\mathrm{II}$ ), treatment with rosiglitazone $4 \mathrm{mg}$ twice daily for 2-6 weeks did not show promising anticancer effect [5]. Similarly, a meta-analysis including 80 randomized clinical trials of rosiglitazone treatment $>24$ weeks did not show a beneficial effect [6]. The summary hazard ratio for incidence of mammary and/or female genital tract malignancies associated with rosiglitazone was 1.19 (95\% confidence interval: 0.62-2.26) [6]. On the other hand, an observational cohort study conducted in France suggested a significantly lower risk of breast cancer associated with rosiglitazone use, with an adjusted hazard ratio of $0.80(95 \%$ confidence interval: $0.73-0.88$ ) [7].

The effects of rosiglitazone and pioglitazone on breast cancer may differ as shown in in vitro and in vivo studies [8]; and in human observational studies [7, 9]. For examples, rosiglitazone but not pioglitazone may induce 
the expression of protein phosphatase and tensin homolog located on chromosome ten, a tumor suppressor gene that may play some role in the pathogenesis of breast cancer [8]. The French study conducted in humans also showed a significantly lower risk associated with rosiglitazone but not with pioglitazone [7]. However, this French study aimed primarily at analyzing the cancer risk associated with pioglitazone and has several limitations such as a restriction of the patients' age within 40-79 years and being able to adjust only for age and other antidiabetic drugs. The investigators did not consider other potential confounders that have been recognized as important risk factors of breast cancer, such as benign breast conditions [10] and use of estrogen [11], and the protective effect of aspirin [12]. Furthermore, the French study failed to consider the effect of differential detection examinations that had been conducted in patients with and without the use rosiglitazone.

In the present study, the association between rosiglitazone and breast cancer was investigated in the Taiwanese female patients with type 2 diabetes mellitus by using the reimbursement database of the National Health Insurance (NHI) from the whole population during the period from 1996 to 2009 .

\section{RESULTS}

Table 1 compares the baseline characteristics between ever users $(n=53029)$ and never users $(n=378418)$ of rosiglitazone. All variables differed significantly. Ever users are characterized by older age, higher proportion with a diabetes duration $\geq 5$ years, higher proportions of all comorbidities and other cancer, higher proportions of using other medications, and a higher proportion of receiving potential detection examinations.

Table 2 shows the incidences of breast cancer in never users, ever users and different subgroups of the dose-response parameters (i.e., the tertile cutoffs of cumulative duration and cumulative dose) of rosiglitazone. The incidence rate in never users and ever users was 249.12 and 217.53 per 100,000 person-years, respectively.

Table 3 shows the hazard ratios with regards to rosiglitazone exposure in different models. The overall hazard ratios for ever users versus never users showed a significantly lower risk in all models. While evaluating the dose-response relationship, a lower risk was observed in the third tertiles of both cumulative duration and cumulative dose in all models and all $P$ values for the trend were significant.

Table 4 shows the joint effects of metformin and rosiglitazone on breast cancer risk. There was a significant interaction between these two drugs. While compared to patients who had not been treated with either drug, users of metformin without rosiglitazone showed a significant $10 \%$ risk reduction, and users of rosiglitazone without metformin showed a non-significant $16 \%$ risk reduction.
The lowest risk was observed in users of both drugs (hazard ratio $0.812,95 \%$ confidence interval: $0.705-0.934$ ).

\section{DISCUSSION}

This is the first study conducted in an Asian population showing that rosiglitazone reduces the risk of breast cancer in Taiwanese female patients with type 2 diabetes mellitus. The findings were consistent in all models and showed an inverse dose-response relationship with both cumulative duration and cumulative dose of therapy (Table 3 ). The reduced risk was statistically significant when the cumulative duration was $>14$ months or when the cumulative dose was $>1792 \mathrm{mg}$ (Table 3). Furthermore, a significant interaction between metformin and rosiglitazone could be observed (Table 4). The lowest risk was seen in patients who used both metformin and rosiglitazone (Table 4).

Such a promising effect provided a rationale for more in-depth investigation on rosiglitazone in the prevention and treatment of breast cancer. Because previous in vitro and in vivo studies [8] and human observational studies [7, 9] suggested that rosiglitazone and pioglitazone may act differently in breast cancer and we have excluded pioglitazone from analyses, whether the beneficial effect of rosiglitazone can be extended to the other thiazolidinediones remains to be answered. Furthermore, the results could not be generalized to nondiabetic individuals, because only patients with type 2 diabetes mellitus were analyzed. Because rosiglitazone rarely induces hypoglycemia when used alone, it will be worthwhile to investigate its effects in nondiabetic women.

Patients with type 2 diabetes mellitus are characterized by insulin resistance $[13,14]$, and female patients with type 2 diabetes mellitus are at an increased risk of breast cancer [15]. Studies suggested that insulin resistance and hyperinsulinemia may play an important role on the development of breast cancer [16, 17]. Adiponectin and leptin are two adipokines that may act oppositely on the risk of breast cancer [18]. While adiponectin may reduce the risk, leptin, on the other hand, is potentially associated with a higher risk [18]. Rosiglitazone and pioglitazone have both been shown to increase the level of adiponectin with little effect on leptin [19, 20]. Therefore, a possible explanation for the beneficial effect of rosiglitazone on breast cancer is through its action on the improvement of insulin resistance by elevating adiponectin levels. However, because both rosiglitazone and pioglitazone increase adiponectin and improve insulin resistance, but only rosiglitazone and probably not pioglitazone may show a beneficial effect on breast cancer risk [7], this may argue against such a mechanism. On the other hand, rosiglitazone may induce the expression of a tumor suppressor gene related to breast cancer but pioglitazone may not [8]. This could partially explain the different clinical effects of rosiglitazone and pioglitazone 
Table 1: Baseline characteristics between never users and ever users of rosiglitazone

\begin{tabular}{|c|c|c|c|c|c|}
\hline \multirow{2}{*}{ Variables } & \multicolumn{2}{|c|}{ Never users } & \multicolumn{2}{|c|}{ Ever users } & \multirow{2}{*}{$\begin{array}{c}P \\
\text { (Chi-square test) }\end{array}$} \\
\hline & $n$ & $\%$ & $n$ & $\%$ & \\
\hline$n=431447$ & 378418 & & 53029 & & \\
\hline \multicolumn{6}{|l|}{ Age (years) } \\
\hline$<40$ & 13108 & 3.46 & 1240 & 2.34 & $<0.0001$ \\
\hline $40-49$ & 37768 & 9.98 & 4495 & 8.48 & \\
\hline $50-59$ & 88584 & 23.41 & 12855 & 24.24 & \\
\hline $60-69$ & 105895 & 27.98 & 16598 & 31.30 & \\
\hline$\geq 70$ & 133063 & 35.16 & 17841 & 33.64 & \\
\hline \multicolumn{6}{|l|}{ Diabetes duration (years) } \\
\hline$<1$ & 30516 & 8.06 & 568 & 1.07 & $<0.0001$ \\
\hline $1-3$ & 58926 & 15.57 & 3002 & 5.66 & \\
\hline $3-4$ & 56427 & 14.91 & 4902 & 9.24 & \\
\hline$\geq 5$ & 232549 & 61.45 & 44557 & 84.02 & \\
\hline Hypertension & 225631 & 59.62 & 41722 & 78.68 & $<0.0001$ \\
\hline Chronic obstructive pulmonary disease & 54417 & 14.38 & 12636 & 23.83 & $<0.0001$ \\
\hline Cerebrovascular disease & 58930 & 15.57 & 14042 & 26.48 & $<0.0001$ \\
\hline Nephropathy & 45488 & 12.02 & 12238 & 23.08 & $<0.0001$ \\
\hline Ischemic heart disease & 89320 & 23.60 & 22117 & 41.71 & $<0.0001$ \\
\hline Peripheral arterial disease & 44918 & 11.87 & 13274 & 25.03 & $<0.0001$ \\
\hline Eye disease & 31704 & 8.38 & 11814 & 22.28 & $<0.0001$ \\
\hline Obesity & 6672 & 1.76 & 1238 & 2.33 & $<0.0001$ \\
\hline Dyslipidemia & 187423 & 49.53 & 36600 & 69.02 & $<0.0001$ \\
\hline Benign breast conditions & 2463 & 0.65 & 471 & 0.89 & $<0.0001$ \\
\hline Other cancer prior to baseline & 42025 & 11.11 & 6351 & 11.98 & $<0.0001$ \\
\hline Sulfonylurea & 272445 & 72.00 & 51200 & 96.55 & $<0.0001$ \\
\hline Metformin & 246129 & 65.04 & 50145 & 94.56 & $<0.0001$ \\
\hline Acarbose & 39774 & 10.51 & 20852 & 39.32 & $<0.0001$ \\
\hline Insulin & 50786 & 13.42 & 21237 & 40.05 & $<0.0001$ \\
\hline Aspirin & 113456 & 29.98 & 28240 & 53.25 & $<0.0001$ \\
\hline Estrogen & 30722 & 8.12 & 7968 & 15.03 & $<0.0001$ \\
\hline Potential detection examinations & 5333 & 1.41 & 971 & 1.83 & $<0.0001$ \\
\hline
\end{tabular}

on breast cancer. The recent study by Cheng et al. pointed to another potential mechanism of rosiglitazone on breast cancer development and growth via the inhibition of a pro-inflammatory and pro-tumor protein Gpr132 in tumor-associated macrophages [4]. Because the effect of pioglitazone on Gpr132 expression has not been examined, it remains unknown whether different drugs in the class of thiazolidinediones may exert different effects on breast cancer risk through their discrepant effects on Gpr132.

The present study confirmed our previous observation of a beneficial effect of metformin on breast cancer [21] (Table 4). Additionally, the present study revealed a significant interaction between metformin and rosiglitazone and the lowest risk was observed in users of both drugs (Table 4).

Because our previous studies suggested that use of insulin [22, 23] and/or sulfonylurea [24] are associated with a significantly higher risk of breast cancer, the higher proportions of their use in ever users of rosiglitazone (Table 1) might have only underestimated the beneficial effect of rosiglitazone.

This study has several strengths. First, potential risk of selection bias related to sampling error was unlikely because the database was derived from the whole population 
Table 2: Incidence of breast cancer by rosiglitazone exposure at entry

\begin{tabular}{lccccc}
\hline Rosiglitazone use & Case number & Incident breast cancer & $\mathbf{\%}$ & Person-years & Incidence rate \\
\cline { 5 - 6 } Never users & 378418 & 3292 & 0.87 & 1321464.67 & 249.12 \\
$\quad$ Ever users & 53029 & 410 & 0.77 & 188477.25 & 217.53 \\
Cumulative duration & (months) & & & & \\
$\quad$ Never users & 378418 & 3292 & 0.87 & 1321464.67 & 249.12 \\
$<3.73$ & 16861 & 138 & 0.82 & 58187.58 & 237.16 \\
$3.73-14$ & 17901 & 138 & 0.77 & 63472.08 & 217.42 \\
$>14$ & 18267 & 134 & 0.73 & 66817.58 & 200.55 \\
Cumulative dose (mg) & & & & & \\
Never users & 378418 & 3292 & 0.87 & 1321464.67 & 249.12 \\
$<448$ & 16845 & 136 & 0.81 & 58281.33 & 233.35 \\
$448-1792$ & 18037 & 141 & 0.78 & 63910.75 & 220.62 \\
$>1792$ & 18147 & 133 & 0.73 & 66285.17 & 200.65 \\
\hline
\end{tabular}

Table 3: Rosiglitazone exposure at entry and hazard ratios for breast cancer in women with type 2 diabetes mellitus

\begin{tabular}{|c|c|c|c|c|c|c|c|c|c|c|c|c|}
\hline \multirow{2}{*}{ Rosiglitazone use } & \multicolumn{3}{|c|}{ Model I } & \multicolumn{3}{|c|}{ Model II } & \multicolumn{3}{|c|}{ Model III } & \multicolumn{3}{|c|}{ Model IV } \\
\hline & HR & $95 \% \mathrm{CI}$ & $P$ value & HR & $95 \%$ CI & $P$ value & HR & $95 \% \mathrm{CI}$ & $P$ value & HR & $95 \%$ CI & $P$ value \\
\hline Ever users vs. never users & 0.854 & $(0.771,0.946)$ & 0.0026 & 0.855 & $(0.772,0.948)$ & 0.0028 & 0.869 & $(0.783,0.964)$ & 0.0081 & 0.889 & $(0.797,0.992)$ & 0.0359 \\
\hline \multicolumn{13}{|l|}{ Cumulative duration (months) } \\
\hline$<3.73$ vs. never users & 0.945 & $(0.797,1.121)$ & 0.5168 & 0.945 & $(0.797,1.121)$ & 0.5159 & 0.966 & $(0.814,1.146)$ & 0.6904 & 0.975 & $(0.818,1.161)$ & 0.7723 \\
\hline $3.73-14$ vs. never users & 0.838 & $(0.707,0.994)$ & 0.0420 & 0.838 & $(0.707,0.994)$ & 0.0422 & 0.860 & $(0.725,1.021)$ & 0.0858 & 0.889 & $(0.746,1.059)$ & 0.1862 \\
\hline$>14$ vs. never users & 0.791 & $(0.666,0.941)$ & 0.0079 & 0.794 & $(0.668,0.944)$ & 0.0090 & 0.795 & $(0.668,0.945)$ & 0.0094 & 0.815 & $(0.682,0.973)$ & 0.0239 \\
\hline$P$-trend & & & 0.0010 & & & 0.0011 & & & 0.0025 & & & 0.0127 \\
\hline \multicolumn{13}{|l|}{ Cumulative dose (mg) } \\
\hline$<448$ vs. never users & 0.926 & $(0.780,1.100)$ & 0.3814 & 0.926 & $(0.780,1.100)$ & 0.3816 & 0.947 & $(0.797,1.124)$ & 0.5318 & 0.908 & $(0.762,1.083)$ & 0.2837 \\
\hline $448-1792$ vs. never users & 0.853 & $(0.720,1.009)$ & 0.0637 & 0.853 & $(0.721,1.010)$ & 0.0652 & 0.877 & $(0.740,1.039)$ & 0.1281 & 0.950 & $(0.799,1.130)$ & 0.5621 \\
\hline$>1792$ vs. never users & 0.793 & $(0.666,0.943)$ & 0.0086 & 0.795 & $(0.668,0.945)$ & 0.0095 & 0.795 & $(0.668,0.946)$ & 0.0097 & 0.815 & $(0.682,0.974)$ & 0.0246 \\
\hline$P$-trend & & & 0.0012 & & & 0.0014 & & & 0.0030 & & & 0.0236 \\
\hline
\end{tabular}

HR: hazard ratio; CI: confidence interval

Model I: unadjusted; Model II: adjusted for age; Model III: adjusted for age, benign breast conditions, use of estrogen and use of aspirin; Model IV: adjusted for all variables in Table 1.

Table 4: Joint effects of metformin and rosiglitazone on breast cancer risk

\begin{tabular}{|c|c|c|c|c|c|c|c|c|}
\hline Metformin/Rosiglitazone use & Case number & $\begin{array}{l}\text { Incident breast } \\
\text { cancer }\end{array}$ & $\%$ & Person-years & $\begin{array}{c}\text { Incidence rate (per 100,000 } \\
\text { person-year) }\end{array}$ & Hazard ratio & $\begin{array}{l}\text { 95\% Confidence } \\
\text { interval }\end{array}$ & $P$ value \\
\hline Metformin (-) / Rosiglitazone (-) & 132613 & 1264 & 0.95 & 460185.58 & 274.67 & 1.000 & & \\
\hline Metformin (+) / Rosiglitazone (-) & 245805 & 2028 & 0.83 & 861279.08 & 235.46 & 0.900 & $(0.817-0.991)$ & 0.0323 \\
\hline Metformin (-) / Rosiglitazone (+) & 2912 & 24 & 0.82 & 10044.33 & 238.94 & 0.836 & $(0.557-1.255)$ & 0.3872 \\
\hline Metformin (+) / Rosiglitazone (+) & 50117 & 386 & 0.77 & 178432.92 & 216.33 & 0.812 & $(0.705-0.934)$ & 0.0036 \\
\hline$P$-interaction & & & & & & & & 0.0434 \\
\hline
\end{tabular}

and it covered the whole period from the beginning of the marketing of rosiglitazone from 2001 in Taiwan to the end of 2009. Second, because the diagnosis of breast cancer was captured from all claim records from outpatient visits and hospital admission, the ascertainment rate should be high. Third, detection rate of breast cancer is less likely to be affected by socioeconomic status because most medical co-payments can be waived in patients with a diagnosis of cancer and there is a low drug cost-sharing in patients with low-income, in veterans or in patients with prescription refills for chronic disease. Fourth, the use of medical records significantly reduced the bias related to self-reporting. 
The study may also suffer from some limitations. First, there was a lack of measurement data for potential confounders such as family history, lifestyle, diet, obesity, tobacco smoking, alcohol drinking, and genetic parameters. Second, no biochemical data were available for evaluating their impacts. These may include hormonal profiles, blood glucose, hemoglobin $\mathrm{A}_{1 \mathrm{C}}$, insulin, $\mathrm{C}$-peptide, or parameters for insulin resistance. Third, we did not have information on the pathology, grading and staging of breast cancer for more in-depth analyses. Fourth, misclassification of breast cancer might occur in some patients. However, such a probability might not be high because mislabeling of a cancer diagnosis in the prescription handed to the patients would not be acceptable when the patients saw the diagnosis.

In summary, this study supports a beneficial effect of rosiglitazone on the prevention of breast cancer in Taiwanese female patients with type 2 diabetes mellitus. Furthermore, a significant interaction between metformin and rosiglitazone can be observed.

\section{MATERIALS AND METHODS}

The study was approved by an ethic review board of the National Health Research Institutes (approval number 99274).

In Taiwan, a compulsory and universal health care system (the so-called NHI) was implemented since March 1995. More than $99 \%$ of the residents are covered by the NHI, and $>98 \%$ of the hospitals throughout Taiwan are under contract with the NHI. Computerized and standard claim documents should be submitted by the contracted medical institutes for reimbursement.

The NHI reimbursement database, which contains detailed records on every visit (outpatient visits and hospital admission) for each patient, is handled by the National Health Research Institutes for academic research. The records include principal and secondary diagnostic codes, prescription orders, and claimed expenses.

Personal information of each individual was deidentified for the protection of privacy. Diabetes was coded 250.XX and breast cancer 174, based on the International Classification of Diseases, Ninth Revision, Clinical Modification (ICD-9-CM).

The database of all patients ever diagnosed of diabetes and under treatment with either oral antidiabetic agents or insulin during 1996-2009 was retrieved from the whole nation. Because pioglitazone may increase the risk of bladder cancer [25-29], patients who had been treated with pioglitazone $(n=235287)$ were first excluded to avoid its contamination. An entry date was set on 1 January 2006. After excluding male patients $(n=935445)$, patients with a diagnosis of diabetes after 2006 ( $n=342351$ ), patients holding a Severe Morbidity Card with a diagnosis of type 1 diabetes $(n=7120$, in Taiwan, a so-called "Severe Morbidity Card" can be issued to patients with type 1 diabetes after certified diagnosis and the patients can be waived of much of the medical co-payments), patients diagnosed of breast cancer before $2006(n=14755)$, patients who died $(n=96320)$ or withdrew from the NHI $(n=12502)$ before entry date, duplicated identification number $(n=106)$, and unclear information on date of birth or sex $(n=5120)$, a total of 431447 female patients diagnosed of type 2 diabetes mellitus and under treatment with oral antidiabetic agents or insulin were included into the analyses.

Patients ever prescribed rosiglitazone before entry date were defined as ever users; and those who had never been treated with rosiglitazone before entry date were defined as never users. The tertile cutoffs of cumulative duration of therapy in months and cumulative dose in $\mathrm{mg}$ were calculated and used as indicators of a dose-response relationship.

An entry date set at the beginning of 2006 was based on the following reasons: 1) Rosiglitazone was marketed in 2001 in Taiwan, and this entry date provided a longest exposure to rosiglitazone for 4 to 5 years at entry and a longest follow-up duration of 4 years; and 2) Pioglitazone was noted to potentially increase the risk of bladder cancer in the PROspective pioglitAzone Clinical Trial In macroVascular Events in 2005 [26], and in 2007, an increased risk of acute myocardial infarction has been challenged with the use of rosiglitazone [30]. These reports had caused tremendous changes in the physicians' prescription behavior and the patients' adherence. The physicians would tend to withdraw thiazolidinediones including rosiglitazone and pioglitazone (troglitazone was not available in Taiwan) and the patients might not adhere to the drugs even if they were prescribed. Therefore, a later entry date after the year 2006 would not only make the estimation of the cumulative duration and cumulative dose of rosiglitazone less reliable, it could also shorten the follow-up duration for cancer incidence.

All comorbidities and covariates were determined as a status/diagnosis before the entry date. The ICD-9-CM codes for the comorbidities were [31-35]: nephropathy 580-589, hypertension 401-405, chronic obstructive pulmonary disease (a surrogate for smoking) 490-496, cerebrovascular disease 430-438, ischemic heart disease 410-414, peripheral arterial disease 250.7, 785.4, 443.81 and 440-448, eye disease 250.5, $362.0,369,366.41$ and 365.44 , obesity 278 , dyslipidemia 272.0-272.4, benign breast conditions $217,610,611,612$, 675,676 , and cancer other than breast cancer 140-208 (excluding 174). Medications included sulfonylurea, metformin, insulin, acarbose, aspirin and estrogen. The following examinations were considered as "potential detection examinations" that might lead to a confounding effect: 1) mammography; and/or 2) breast sonography. Baseline characteristics were compared by Chi-square test between ever users and never users of rosiglitazone.

The incidence density of breast cancer was calculated for different subgroups of rosiglitazone 
exposure including ever users, never users and the tertiles of cumulative duration and cumulative dose. The numerator was the number of patients with incident breast cancer during follow-up, and the denominator was the person-years of follow-up. For ever users, the follow-up ended on the date of breast cancer diagnosis or on the date of the last record in the reimbursement database in individuals without incident breast cancer. For never users, the follow-up ended on the date of breast cancer diagnosis, or on the date of rosiglitazone initiation or the last reimbursement record, depending on which occurring first. This would ensure no exposure to rosiglitazone at the end of follow-up in the never users.

Cox proportional hazards regression was used to estimate the hazard ratios for breast cancer for ever users and for the tertiles of cumulative duration and cumulative dose, using never users as the referent group. The following four models were created: 1) unadjusted; 2) adjusted for age; 3 ) adjusted for selected important risk factors of breast cancer including age, benign breast conditions, use of estrogen, and use of aspirin; and 4) adjusted for all baseline characteristics (fully adjusted).

Metformin has been shown to reduce the risk of various types of cancer [36-42]. Because our previous study also suggested that metformin may prevent breast cancer [21], additional analyses were conducted to evaluate the potential joint effects of metformin and rosiglitazone. The incidence rates of breast cancer and the fully adjusted hazard ratios were calculated for the following 4 subgroups with regards to the use of metformin and rosiglitazone: 1) metformin (-)/rosiglitazone (-) as referent, 2) metformin $(+)$ /rosiglitazone $(-), 3)$ metformin $(-)$ /rosiglitazone $(+)$, and 4) metformin $(+)$ /rosiglitazone $(+)$.

Analyses were conducted using SAS statistical software, version 9.3 (SAS Institute, Cary, NC). $P<0.05$ was considered statistically significant.

\section{ACKNOWLEDGMENTS}

The study is based in part on data from the National Health Insurance Research Database provided by the Bureau of National Health Insurance, Department of Health and managed by National Health Research Institutes. The interpretation and conclusions contained herein do not represent those of Bureau of National Health Insurance, Department of Health or National Health Research Institutes.

\section{CONFLICTS OF INTEREST}

The author declares no conflicts of interest.

\section{FUNDINGS}

The study was supported by the Ministry of Science and Technology (MOST 103-2314-B-002-187-MY3) of
Taiwan. The funders had no role in study design, data collection and analysis, decision to publish, or preparation of the manuscript. The publication fee of this paper is supported by the Weng Yuan Endocrine Fund.

\section{REFERENCES}

1. Kotta-Loizou I, Giaginis C, Theocharis S. The role of peroxisome proliferator-activated receptor- $\gamma$ in breast cancer. Anticancer Agents Med Chem. 2012; 12:1025-44.

2. Bojková B, Garajová M, Kajo K, Péc M, Kubatka P, Kassayová M, Kisková T, Orendás P, Ahlersová E, Ahlers I. Pioglitazone in chemically induced mammary carcinogenesis in rats. Eur J Cancer Prev. 2010; 19:379-84.

3. Kocdor H, Kocdor MA, Canda T, Gurel D, Cehreli R, Yilmaz O, Alakavuklar M, Guner G. Chemopreventive efficacies of rosiglitazone, fenretinide and their combination against rat mammary carcinogenesis. Clin Transl Oncol. 2009; 11:243-9.

4. Cheng WY, Huynh H, Chen P, Peña-Llopis S, Wan Y. Macrophage PPAR $\gamma$ inhibits Gpr132 to mediate the antitumor effects of rosiglitazone. Elife. 2016; 3:5.

5. Hatton JL, Yee LD. Clinical Use of PPARgamma Ligands in Cancer. PPAR Res. 2008; 2008:159415.

6. Monami M, Lamanna C, Marchionni N, Mannucci E. Rosiglitazone and risk of cancer: a meta-analysis of randomized clinical trials. Diabetes Care. 2008; 31:1455-60.

7. Neumann A, Weill A, Ricordeau P, Fagot JP, Alla F, Allemand H. Pioglitazone and risk of bladder cancer among diabetic patients in France: a population-based cohort study. Diabetologia. 2012; 55:1953-62.

8. Teresi RE, Shaiu CW, Chen CS, Chatterjee VK, Waite KA, Eng C. Increased PTEN expression due to transcriptional activation of PPARgamma by lovastatin and rosiglitazone. Int J Cancer. 2006; 118:2390-8.

9. Ferrara A, Lewis JD, Quesenberry CP Jr, Peng T, Strom BL, Van Den Eeden SK, Ehrlich SF, Habel LA. Cohort study of pioglitazone and cancer incidence in patients with diabetes. Diabetes Care. 2011; 34:923-9.

10. Gadducci A, Guerrieri ME, Genazzani AR. Benign breast diseases, contraception and hormone replacement therapy. Minerva Ginecol. 2012; 64:67-74.

11. Chlebowski RT, Anderson GL. Changing concepts: Menopausal hormone therapy and breast cancer. J Natl Cancer Inst. 2012; 104:517-27.

12. Bardia A, Olson JE, Vachon CM, Lazovich D, Vierkant RA, Wang AH, Limburg PJ, Anderson KE, Cerhan JR. Effect of aspirin and other NSAIDs on postmenopausal breast cancer incidence by hormone receptor status: results from a prospective cohort study. Breast Cancer Res Treat. 2011; 126:149-55.

13. Kashyap SR, Defronzo RA. The insulin resistance syndrome: physiological considerations. Diab Vasc Dis Res. 2007; 4:13-9. 
14. Arcidiacono B, Iiritano S, Nocera A, Possidente $K$, Nevolo MT, Ventura V, Foti D, Chiefari E, Brunetti A. Insulin resistance and cancer risk: an overview of the pathogenetic mechanisms. Exp Diabetes Res. 2012; 2012:789174.

15. Tseng CH, Chong CK, Tai TY. Secular trend for mortality from breast cancer and the association between diabetes and breast cancer in Taiwan between 1995 and 2006 . Diabetologia. 2009; 52:240-6.

16. Xue F, Michels KB. Diabetes, metabolic syndrome, and breast cancer: a review of the current evidence. Am J Clin Nutr. 2007; 86:823S-5S.

17. Kabat GC, Kim M, Caan BJ, Chlebowski RT, Gunter MJ, Ho GY, Rodriguez BL, Shikany JM, Strickler HD, Vitolins MZ, Rohan TE. Repeated measures of serum glucose and insulin in relation to postmenopausal breast cancer. Int J Cancer. 2009; 125:2704-10.

18. Esper RM, Dame M, McClintock S, Holt PR, Dannenberg AJ, Wicha MS, Brenner DE. Leptin and adiponectin modulate the self-renewal of normal human breast epithelial stem cells. Cancer Prev Res (Phila). 2015; 8:1174-83.

19. Oz O, Tuncel E, Eryilmaz S, Fazlioglu M, Gul CB, Ersoy C, Ocak N, Dirican M, Cangur S, Baran I, Imamoglu S. Arterial elasticity and plasma levels of adiponectin and leptin in type 2 diabetic patients treated with thiazolidinediones. Endocrine. 2008; 33:101-5.

20. Miyazaki Y, DeFronzo RA. Rosiglitazone and pioglitazone similarly improve insulin sensitivity and secretion, glucose tolerance and adipocytokines in type 2 diabetic patients. Diabetes Obes Metab. 2008; 10:1204-11.

21. Tseng $\mathrm{CH}$. Metformin may reduce breast cancer risk in Taiwanese women with type 2 diabetes. Breast Cancer Res Treat. 2014; 145:785-90.

22. Tseng $\mathrm{CH}$. Use of insulin and mortality from breast cancer among Taiwanese women with diabetes. J Diabetes Res. 2015; 678756.

23. Tseng CH. Prolonged use of human insulin increases breast cancer risk in Taiwanese women with type 2 diabetes. BMC Cancer. 2015; 15:846.

24. Hsieh MC, Lee TC, Cheng SM, Tu ST, Yen MH, Tseng CH. The influence of type 2 diabetes and glucose-lowering therapies on cancer risk in the Taiwanese. Exp Diabetes Res. 2012; 2012:413782.

25. Lewis JD, Ferrara A, Peng T, Hedderson M, Bilker WB, Quesenberry CP Jr, Vaughn DJ, Nessel L, Selby J, Strom BL. Risk of bladder cancer among diabetic patients treated with pioglitazone: interim report of a longitudinal cohort study. Diabetes Care. 2011; 34:916-22.

26. Dormandy JA, Charbonnel B, Eckland DJ, Erdmann E, Massi-Benedetti M, Moules IK, Skene AM, Tan MH, Lefèbvre PJ, Murray GD, Standl E, Wilcox RG, Wilhelmsen L, et al. PROactive investigators. Secondary prevention of macrovascular events in patients with type 2 diabetes in the PROactive Study (PROspective pioglitAzone Clinical Trial In macroVascular Events): a randomised controlled trial. Lancet. 2005; 366:1279-89.
27. Tseng $\mathrm{CH}$. Pioglitazone and bladder cancer in human studies: is it diabetes itself, diabetes drugs, flawed analyses or different ethnicities? J Formos Med Assoc. 2012; 111:123-31.

28. Tseng $\mathrm{CH}$. A review on thiazolidinediones and bladder cancer in human studies. J Environ Sci Health C Environ Carcinog Ecotoxicol Rev. 2014; 32:1-45.

29. Tseng $\mathrm{CH}$, Tseng FH. Peroxisome proliferator-activated receptor agonists and bladder cancer: lessons from animal studies. J Environ Sci Health C Environ Carcinog Ecotoxicol Rev. 2012; 30:368-402.

30. Nissen SE, Wolski K. Effect of rosiglitazone on the risk of myocardial infarction and death from cardiovascular causes. N Engl J Med. 2007; 356:2457-71.

31. Tseng $\mathrm{CH}$. Pioglitazone and bladder cancer: a populationbased study of Taiwanese. Diabetes Care. 2012; 35:278-80.

32. Tseng $\mathrm{CH}$. Benign prostatic hyperplasia is a significant risk factor for bladder cancer in diabetic patients: a populationbased cohort study using the National Health Insurance in Taiwan. BMC Cancer. 2013; 13:7.

33. Tseng $\mathrm{CH}$. Thyroid cancer risk is not increased in diabetic patients. PLoS One. 2012; 7:e53096.

34. Tseng CH. Diabetes, metformin use, and colon cancer: A population-based cohort study in Taiwan. Eur J Endocrinol. 2012; 167:409-16.

35. Tseng $\mathrm{CH}$. Diabetes and risk of bladder cancer: A study using the National Health Insurance database in Taiwan. Diabetologia. 2011; 54:2009-15.

36. Chae YK, Arya A, Malecek MK, Shin DS, Carneiro B, Chandra S, Kaplan J, Kalyan A, Altman JK, Platanias L, Giles F. Repurposing metformin for cancer treatment: current clinical studies. Oncotarget. 2016; 7:40767-80. doi: 10.18632/oncotarget.8194.

37. Ma SJ, Zheng YX, Zhou PC, Xiao YN, Tan HZ. Metformin use improves survival of diabetic liver cancer patients: systematic review and meta-analysis. Oncotarget. 2016; 7:66202-66211. doi: 10.18632/oncotarget.11033.

38. Tseng $\mathrm{CH}$. Metformin use and cervical cancer risk in female patients with type 2 diabetes. Oncotarget. 2016; 7:5954859555. doi: 10.18632/oncotarget.10934.

39. Poli G, Cantini G, Armignacco R, Fucci R, Santi R, Canu L, Nesi G, Mannelli M, Luconi M. Metformin as a new anticancer drug in adrenocortical carcinoma. Oncotarget. 2016; 7:49636-49648. doi: 10.18632/oncotarget.10421.

40. Menendez JA, Martin-Castillo B, Joven J. Metformin and cancer: Quo vadis et cui bono? Oncotarget. 2016; 7:5409654101. doi: 10.18632/oncotarget.10262.

41. Tseng $\mathrm{CH}$. Metformin may reduce oral cancer risk in patients with type 2 diabetes. Oncotarget. 2016; 7:2000-8. doi: 10.18632/oncotarget.6626.

42. Anisimov VN. Metformin for cancer and aging prevention: is it a time to make the long story short? Oncotarget. 2015; 6:39398-407. doi: 10.18632/oncotarget.6347. 